\title{
Superspace Ward Identities in Supersymmetric Gauge Theories
}

\author{
Asim Gangopadhyaya \\ Loyola University Chicago, agangop@luc.edu \\ Darwin Chang \\ University of Maryland - College Park
}

Follow this and additional works at: https://ecommons.luc.edu/physics_facpubs

Part of the Other Physics Commons, and the Quantum Physics Commons

\section{Recommended Citation}

Chang, D. and Gangopadhyaya, A. (1984). Superspace ward identities in supersymmetric gauge theories. Pramana, 23(5), 631-637.

This Article is brought to you for free and open access by the Faculty Publications and Other Works by Department at Loyola eCommons. It has been accepted for inclusion in Physics: Faculty Publications and Other Works by an authorized administrator of Loyola eCommons. For more information, please contact ecommons@luc.edu.

\section{(c) $(\mathrm{i})($ )}

This work is licensed under a Creative Commons Attribution-Noncommercial-No Derivative Works 3.0 License. (C) Springer India, 1984. 
Pramāṇa, Vol. 23, No. 5, November 1984, pp. 631-637. (C) Printed in India.

\title{
Superspace Ward identities in supersymmetric gauge theories
}

\author{
DARWIN CHANG and ASIM GANGOPADHYAYA* \\ Department of Physics and Astronomy, University of Maryland, College Park, MD 20742 \\ * Physics Department, City College of New York, New York, NY 10031 \\ MS received 26 June 1984
}

\begin{abstract}
In superspace formulation of supersymmetric gauge theories, gauge invariance requires an infinite set of identities between the infinite set of renormalization constants. Using Ward identities in superspace, the same is derived. These identities at one loop level are also
demonstrated.
\end{abstract}

Keywords. Superspace; Ward identities; gauge theories; renormalization.

PACS No. ' $11 \cdot 30 \mathrm{~Pb}^{\prime}$

\section{Introduction}

The supersymmetric (SUSY) versions of both abelian and non-abelian gauge theories were formulated some time ago (Salam and Strathdee 1974; Wess and Zumino 1974; Ferrara and Zumino 1974; Ferrara et al 1974; Ovrut and Wess 1982). In the original component formalism, the SUSY gauge theory, demonstrates a dramatic cancellation of divergences between the fermionic and bosonic contributions such that the divergent structure of the theory is greatly reduced (Wess and Bagger 1983; Gates et al 1983) review. The theory can be formulated more schematically in terms of superfields, residing in superspace (Salam and Strathdee 1974; Ferrara et al 1974) of space-time and grassmanian coordinates, which serve as representations of the supersymmetric transformation. From the superfield point of view, those seemingly miraculous cancellations in the component formalism are automatic consequences of the very existence of superfields in superspace. In this sense, the superfield formalism is a more natural description of the susy theories. Since their invention, the path integral quantization and Feynman rules have been worked out in literature (Salam and Strathdee 1975; Grisaru et al 1979). In addition to being a conceptual tool, the superfield method has attained increasing attention in application to practical calculation. In this paper, we take a look at the SUSY gauge theory from the superfield point of view.

In sUSY gauge theory, the gauge field is contained in a vector supermultiplet. The vector multiplet contains, besides gauge boson and gaugino fields, many (super-) gauge degrees of freedom. In component language, in order to remove these spurious degrees of freedom and simplify the formalism, a particular gauge, called the Wess-Zumino gauge has to be chosen (Wess and Zumino 1974). This choice of gauge explicitly breaks the supersymmetric invariance and makes the maintenance of supersymmetry at higher order a nontrivial task. Such a complication can be avoided altogether if one adopts the superfield approach. However, the advantage one obtains in this aspect is not without 